\title{
Sistema de freqüência modulada no transtorno do processamento auditivo: prática baseada em evidências? $* * * * * * *$
}

\author{
Isabel Cristina Cavalcanti Lemos* \\ Regina Tangerino de Souza Jacob** \\ Mariana Germano Gejão*** \\ Maria Cecília Bevilacqua**** \\ Mariza Ribeiro Feniman***** \\ Deborah Viviane Ferrari******
}

\author{
Frequency modulation (FM) system in auditory \\ processing disorder: an evidence-based practice?
}

*Fonoaudióloga. Mestre em Fonoaudiologia pela Faculdade de Odontologia de Bauru (FOB) Universidade de São Paulo (USP). Fonoaudióloga da Associação de Pais e Amigos dos Deficiêntes Auditivos de Sorocaba (Apadas). Endereço para correspondência: Rua Bartolome Bueno, 155 - Apto. 32 - Sorocaba - São Paulo - CEP 18065-255 (bel_ccl@yahoo.com.br).

**Fonoaudióloga. Doutora em Ciências da Reabilitação - Distúrbios da Comunicação Humana pelo Hospital de Reabilitação de Anomalias Craniofaciais (HRAC) da USP - Bauru. Professora Doutora do Departamento de Fonoaudiologia da FOB - USP.

***Fonoaudióloga. Mestre em Fonoaudiologia pela FOB - USP. Fonoaudióloga da Clínica de Fonoaudiologia da FOB - USP

****Fonoaudióloga. Professora Titula do Departamento de Fonoaudiologia da FOB - USP. Coordenadora do Centro de Pesquisas Audiológicas do HRAC da USP - Campus Bauru.

*****Fonoaudióloga. Professora Livre Docente do Departamento de Fonoaudiologia da FOB - USP.

******Fonoaudióloga. Doutora em Neurociências e Comportamento pelo Instituto de Psicologia - USP. Professora Doutora do Departamento de Fonoaudiologia da FOB - USP.

*******Trabalho Realizado no Departamento de Fonoaudiologia da FOB - USP.

Artigo de Revisão de Literatura e Revisão Sistemática.

Artigo Submetido a Avaliação por Pares Conflito de Interesse: não

Recebido em 27.11.2008.

Revisado em 06.04.2009; 10.07.2009.

Aceito para Publicação em 10.07.2009.

Referenciar este material como:

Lemos ICC, Jacob RTS, Gejão MG, Bevilacqua MC, Feniman MR, Ferrari DV. Sistema de freqüência modulada no transtorno do processamento auditivo: prática baseada $\sum 3$ em evidências. Pró-Fono Revista de Atualização Científica. 2009 jul-set;21(3):243-8.

\begin{abstract}
Background: use of frequency modulation (FM) system in auditory processing disorder. Aim: to verify the existence of scientific evidence confirming the effectiveness of personal FM systems in the treatment of central auditory processing disorders (APD). For this purpose a systematic review of the literature was made, using data found in electronic databases (Medline, Lilacs and Cochrane library) as well as on the internet. The articles retrieved were analyzed according to the CONSORT statement and then classified by their evidence level. Conclusion: the search resulted in 1,589 references out of which only 19 met the inclusion criteria. All of the analyzed articles were classified as having low level of evidence (expert opinion or case studies). Strong scientific evidence supporting the use of personal FM systems for APD intervention was not found. Since such device is frequently recommended for the treatment of APD, it becomes essential to carry out studies with high scientific evidence that could safely guide clinical decision making on this subject.
\end{abstract}

Key Words: Central Auditory Diseases; Hearing Aids; Rehabilitation; Audiology.

\section{Resumo}

Tema: uso do sistema de frequência modulada (FM) no transtorno do processamento auditivo. Objetivo: verificar a existência de evidência científica que comprove a efetividade do sistema FM para tratamento do transtorno do processamento auditivo (TPA) por meio de revisão sistemática da literatura pertinente encontrada em bases de dados eletrônicas (Medline, Lilacs e registro de ensaios clínicos da biblioteca Cochrane) e buscadores da internet, sendo os artigos recuperados analisados com base na declaração CONSORT e classificados quanto ao nível de evidência. Conclusão: a busca resultou em 1589 referências, das quais somente 19 enquadraram-se nos critérios de inclusão. Todos os artigos analisados foram classificados com baixo nível de evidência (opinão de especialista ou estudo de casos). Não foi encontrada forte evidência científica que comprove a efetividade do uso do sistema FM na intervenção de indivíduos com TPA. Uma vez que este equipamento é freqüentemente recomendado para a intervenção nos casos do TPA, se torna imprescindível a realização de estudos com alta evidência científica que possam guiar seguramente as tomadas de decisões clínicas a este respeito.

Palavras-Chave: Doenças Auditivas Centrais; Auxiliares de Audição; Reabilitação; Audiologia. 


\section{Introdução}

Indivíduos com transtorno do processamento auditivo (TPA) apresentam uma ou mais das seguintes características comportamentais: dificuldade em compreender a linguagem em ambientes com ruído competitivo ou reverberante, respostas inconsistentes ou inapropriadas para a fala, solicitação freqüente de repetição da mensagem falada, dificuldade atencional, facilidade de distração, dificuldade em seguir ordens verbais complexas, dificuldade em localizar o som, dificuldade em aprender músicas e ritmos e problemas de leitura e aprendizagem. O TPA freqüentemente exerce maior impacto em crianças, podendo tais características interferir nas habilidades sociais e de leitura, fala, linguagem, compreensão, atenção e comunicação e, desta forma, gerar ansiedade, baixa auto-estima e desempenho acadêmico aquém do esperado ${ }^{1-2}$.

As crianças passam muito de seu tempo na escola e em 45\% desse tempo estão envolvidas em atividades nas quais predomina a fala dos professores e de seus colegas. Deste modo, é fácil concluir que a clara recepção da mensagem e capacidade de processá-la auditivamente é essencial para a aprendizagem escolar ${ }^{3}$.

A mensagem falada é altamente redundante. No contexto diário de comunicação ouvintes sem alterações nas vias auditivas periféricas e centrais não necessitam de todas as pistas acústicas contidas em uma mensagem para compreendê-la. Por outro lado, indivíduos com alterações auditivas terão mais dificuldade em compreender a mensagem se a diminuição de tais pistas ocorrer.

A eliminação das informações redundantes da fala ocasionada pela presença do ruído, da reverberação e da distância entre falantes tem um efeito severo na percepção da fala principalmente em indivíduos com necessidades auditivas especiais como, por exemplo, crianças com comprometimento de fala e linguagem, do processamento auditivo ou com distúrbio de aprendizagem. Desta forma, ao longo do dia o aluno terá despendido grande energia para lidar com as situações acústicas adversas que podem levar à exaustão e, conseqüentemente, baixo rendimento escolar e pouco aproveitamento do aprendizado. Por esta razão a perda adicional de informações de fala deve ser evitada ao máximo. É importante ressaltar que as habilidades auditivas, atenção e memória se tornarão semelhantes à de um adulto por volta dos 12 anos de idade ${ }^{3}$.

O sistema de freqüência modulada (FM) consiste de dois elementos: um microfone/ transmissor e um receptor. O microfone/transmissor, utilizado pelo falante, capta os sons, os codifica em sinais elétricos e depois os converte em sinais de freqüência modulada. Este sinal FM é decodificado pelo receptor, utilizado pelo ouvinte, sendo novamente transformado em energia acústica. A transmissão FM propicia uma solução simples para reduzir a distância entre falante e ouvinte e, por conseqüência, diminuir o efeito mascarado do ruído e da reverberação sobre o sinal de fala ${ }^{4}$.

Por esta atuação, o sistema FM pode ser considerado como mais uma alternativa dentre os materiais diferenciados e recursos de apoio utilizados por alunos portadores de necessidades educativas especiais, visando a integração educativo-escolar ${ }^{5}$.

O sistema FM pessoal também pode ser indicado para crianças sem perda auditiva periférica, mas que apresentam dificuldades de aprendizagem associadas a déficit de atenção $0^{4}$ e com desordens do processamento auditivo central ${ }^{6-7}$. Para essa população, uma favorável relação $S / R$ parece facilitar a atenção para as tarefas e melhorar o tempo de resposta, pois quando a fala do professor se torna mais clara, as crianças apresentam um tempo maior de foco e concentração no estímulo sonoro relevante, enquanto ignoram o estímulo competitivo.

Atualmente muito tem sido discutido a respeito da prática baseada em evidência - definida como o uso claro e ponderado das melhores evidências científicas existentes na decisão sobre o cuidado de pacientes, integrando a experiência com as melhores evidências clínicas obtidas em pesquisa sistemática. Neste contexto, prioridade é dada para resultados de pesquisas clínicas originais especialmente planejadas e com suporte metodológico específico e rigoroso, que realmente mensuram o sucesso de tratamentos em condições reais, ficando os livros e opiniões de especialistas com menor importância ${ }^{8-9}$. Portanto, se tornam imprescindíveis a preocupação e realização de estudos com evidência científica que possam guiar seguramente as tomadas de decisões clínicas a este respeito, visto que na Fonoaudiologia é comum que os dados científicos disponíveis não apresentem um nível forte de evidência que acompanhem o sucesso observado na prática clínica ${ }^{10}$.

Diante de indicações do uso do sistema FM pessoal para indivíduos com o TPA, o presente estudo teve como objetivo realizar a revisão sistemática da literatura a fim de verificar a existência de evidências científicas que sustentem esta prática clínica.

Para o desenvolvimento deste estudo, foram realizadas buscas da literatura pertinente em bases de dados eletrônicas: 
. Centro Latino-Americano e do Caribe de Informação em Ciências da Saúde - Bireme (www.bireme.br): base de dados Lilacs (Literatura em Ciências da Saúde publicada na América Latina e Caribe, a partir de 1982) e Medline (Literatura Internacional da Área Médica e Biomédica, compilados desde 1966); . Pubmed (www.pubmed.com): serviço da National Library of Medicine e National Institute of Health, inclui citações da Medline e outros periódicos da área de "ciências da vida", compilados desde 1948; . Eletronic Reference Library (www.portal dapesquisa.com.br): acesso a mais de 30 bases de dados, dentre elas as bases ERIC (educação) e PsycInfo (psicologia, educação, psiquiatria, ciências sociais); . Web of Science (www.thomsonisi.com): Base de dados áreas das ciências, ciências sociais, artes e humanidades;

. Biblioteca Cochrane (http://cochrane.bvsalud.org): acesso às bases de dados Cochrane de revisões sistemáticas, registro de ensaios controlados da Colaboração Cochrane, avaliação de tecnologias em saúde, entre outras.

TABELA 1. Relação das palavras e descritores de assunto utilizados nas bases de dados e ferramentas de busca.

\begin{tabular}{c|c}
\hline Número da Busca & Palavras e Descritores Cruzados \\
\hline 1 & auditory AND processing AND personal AND FM \\
2 & auditory AND processing AND FM AND technolog* \\
3 & auditory AND processing AND FM AND system* \\
4 & auditory AND processing AND frequency modula* \\
5 & auditory diseases, central AND personal AND FM \\
6 & auditory diseases, central AND FM AND technolog* \\
7 & auditory diseases, central AND FM AND system* \\
8 & auditory diseases, central AND frequency modula* \\
\hline
\end{tabular}

* palavras truncadas conforme cada base de dados.
Também foram utilizadas ferramentas de buscas como o Google Acadêmico (www.scholar.google.com) e buscas manuais a partir das referências bibliográficas dos artigos encontrados nas bases acima descritas e recuperados.

Foram utilizados descritores extraídos do Medical Subject Headings (MeSH) e sua respectiva (DeCS) e palavras chaves utilizadas nas bases de dados foram cruzados conforme descrito na Tabela 1.

A busca não foi limitada por ano, portanto, os resultados englobam todos os artigos que constavam em cada uma das bases de dado desde o seu início até abril de 2008. Não houve restrição quanto à língua em que os trabalhos foram publicados.

\section{Critérios de inclusão da literatura}

Foram utilizados como critério de inclusão:

. ensaios clínicos randomizados controlados que pudessem evidenciar o uso do sistema FM no tratamento dos TPA;

. estudos que abordassem no título e/ou resumo formas de intervenção nos TPA e que citassem o uso do sistema FM pessoal em alguma parte do texto; . estudos com indivíduos que apresentassem audição periférica normal.

Análise dos resultados

A despeito da estratégia de busca utilizada (Tabela 1) a pesquisa na biblioteca Cochrane revelou inexistência de revisões sistemáticas ou ensaios clínicos registrados neste tema.

Nas demais bases de dados a busca resultou em 1589 citações (Tabela 2). Apartir da análise dos resumos foram excluídas as citações repetidas e aquelas que não se enquadravam nos critérios de recuperação.

TABELA 2. Relação dos estudos encontrados em cada bases de dados, de acordo com cada número de busca.

\begin{tabular}{|c|c|c|c|c|c|c|c|c|c|c|c|c|c|c|c|c|}
\hline \multirow[t]{2}{*}{ Número da Busca } & \multicolumn{2}{|c|}{$\begin{array}{l}\text { LILACS } \\
\text { (Bireme) }\end{array}$} & \multicolumn{2}{|c|}{$\begin{array}{c}\text { MEDLINE } \\
\text { (Bireme) }\end{array}$} & \multicolumn{2}{|c|}{ Pubmed } & \multicolumn{2}{|c|}{$\begin{array}{l}\text { ERIC } \\
(E R L)\end{array}$} & \multicolumn{2}{|c|}{$\begin{array}{c}\text { MEDLINE } \\
\text { (ERL) }\end{array}$} & \multicolumn{2}{|c|}{$\begin{array}{c}\text { PsychoInfo } \\
\text { (ERL) }\end{array}$} & \multicolumn{2}{|c|}{ Web of Science } & \multicolumn{2}{|c|}{ Google Scholar } \\
\hline & $\mathrm{E}$ & $\mathrm{I}$ & $\mathrm{E}$ & I & $\mathrm{E}$ & I & $\mathrm{E}$ & I & $\mathrm{E}$ & $\mathrm{I}$ & $\mathrm{E}$ & I & $\mathrm{E}$ & I & $\mathrm{E}$ & I \\
\hline 1 & - & - & 1 & 1 & - & - & - & - & - & - & - & - & - & - & $* *$ & $* *$ \\
\hline 2 & - & - & - & - & - & - & - & - & 5 & - & - & - & - & - & $* *$ & $* *$ \\
\hline 3 & - & - & 30 & 1 & 22 & - & - & - & 3 & - & 15 & 1 & 41 & - & $* *$ & $* *$ \\
\hline 4 & 1 & - & 243 & 7 & - & - & - & - & 348 & - & 152 & - & 414 & - & 314 & 10 \\
\hline 5 & - & - & - & - & - & - & - & - & - & - & - & - & $*$ & $*$ & $*$ & $*$ \\
\hline 6 & - & - & - & - & - & - & - & - & - & - & - & - & $*$ & $*$ & $*$ & $*$ \\
\hline 7 & - & - & - & - & - & - & - & - & - & - & - & - & * & $*$ & $*$ & $*$ \\
\hline 8 & - & - & - & - & - & - & - & - & - & - & - & - & $*$ & $*$ & $*$ & $*$ \\
\hline
\end{tabular}

Legenda: - = nenhum estudo encontrado; * = não utiliza busca por descritor de assunto; ** = não foi realizada busca com tais palavras; E = estudos encontrados; I = estudos incluídos na análise. 
Destas somente 20 enquadraram-se nos critérios de inclusão.

Três revisores independentes avaliaram os artigos recuperados, classificando-os de acordo com os níveis de evidência ${ }^{9}$ descritos na Tabela 3. Para fins de caracterização dos estudos clínicos randomizados controlados optou-se pela utilização das recomendações CONSORT (Consolidated Standards of Reporting Trials ${ }^{11}$. As análises de cada revisor foram então comparadas. Reuniões de consenso resolveram discordância entre os dados, quando necessário.
TABELA 3. Descrição dos seis níveis de evidência de um estudo.

\begin{tabular}{c|c}
\hline $\begin{array}{c}\text { Nível de } \\
\text { Evidência }\end{array}$ & Descrição \\
\hline 1 & revisões sistemáticas e meta-análise de estudos clínicos \\
randomizados e controlados \\
2 & estudos clínicos randomizados e controlados \\
3 & estudos de intervenções não randomizados \\
4 & estudos descritivos (estudos transversais, estudos de coorte, \\
& casos-controle) \\
5 & estudos de casos \\
6 & opinião de especialistas \\
\hline
\end{tabular}

Fonte: Cox .

TABELA 4. Descrição dos trabalhos analisados segundo nome dos autores, título, ano, design e nível de evidência.

\begin{tabular}{|c|c|c|c|c|}
\hline Autor & Título & Ano & Design & $\begin{array}{l}\text { Nível de } \\
\text { Evidência }\end{array}$ \\
\hline ASHA $^{24}$ & (Central) Auditory processing disorders & 2005b & opinião de especialista & 6 \\
\hline Baran JA ${ }^{16}$ & $\begin{array}{l}\text { Managing Auditory Processing Disorders in Adolescents and } \\
\text { Adults }\end{array}$ & 2002 & opinião de especialista & 6 \\
\hline Beck DL; Bellis TJ ${ }^{18}$ & $\begin{array}{l}\text { (Central) auditory processing disorders: } \\
\text { Overview and amplification issues }\end{array}$ & 2007 & opinião de especialista & 6 \\
\hline Bellis $\mathrm{TJ}^{13}$ & $\begin{array}{c}\text { Developing Deficit-Specific Intervention Plans for Individuals with } \\
\text { Auditory Processing Disorders }\end{array}$ & 2002 & opinião de especialista & 6 \\
\hline Bellis TJ; Ferre $\mathrm{JM}^{29}$ & $\begin{array}{l}\text { Multidimensional Approach to the differential diagnosis of central } \\
\text { auditory processing disorders in children }\end{array}$ & 1999 & estudo de casos & 5 \\
\hline Cameron S; Dillon $\mathrm{H}^{30}$ & $\begin{array}{l}\text { The listening in spatialized noise-sentences test } \\
\text { (LISN-S): test-retest reliability study }\end{array}$ & 2007 & opinião de especialista & 6 \\
\hline Ferre $\mathrm{JM}^{17}$ & $\begin{array}{l}\text { Managing Children's Central Auditory Processing Deficits in the } \\
\text { Real World: What Teachers and Parents Want to Know }\end{array}$ & 2002 & opinião de especialista & 6 \\
\hline Flexer $\mathrm{C}^{19}$ & The impact of classroom acoustics: listening, learning and literacy & 2004 & opinião de especialista & 6 \\
\hline Phonak $^{27}$ & $\begin{array}{c}\text { Ear-level FM receiver stimulates auditory neural plasticity in } \\
\text { children with APD }\end{array}$ & 2006 & $\begin{array}{l}\text { estudos descritivos } \\
\text { (casos controle) }\end{array}$ & 4 \\
\hline Friel-Patti ${ }^{14}$ & $\begin{array}{l}\text { Clinical decision-making in the assessment and intervention of } \\
\text { central auditory processing disorders }\end{array}$ & 1999 & opinião de especialista & 6 \\
\hline Keith RW ${ }^{23}$ & Clinical issues in central auditory processing disorders & 1999 & opinião de especialista & 6 \\
\hline Kreisman $\mathrm{BM}^{26}$ & $\begin{array}{c}\text { Frequency Modulation (FM) Systems for children with normal } \\
\text { hearing }\end{array}$ & 2002 & $\begin{array}{l}\text { estudos descritivos } \\
\text { (estudo coorte) }\end{array}$ & 4 \\
\hline Launer $\mathrm{S}^{15}$ & $\begin{array}{l}\text { Wireless solutions: the state of the art and future of FM technology } \\
\text { for hearing impaired consumer }\end{array}$ & 2004 & opinião de especialista & 6 \\
\hline Rosenberger GG ${ }^{20}$ & $\begin{array}{c}\text { Classroom acoustics and personal FM technology in manegment of } \\
\text { auditory processing disorder }\end{array}$ & 2002 & opinião de especialista & 6 \\
\hline Sharma M; Purdy SC ${ }^{28}$ & A case study of an 11-Year-Old with auditory processing disorder & 2007 & estudo de caso & 5 \\
\hline $\begin{array}{l}\text { Souza-Jacob RT, } \\
\text { Almeida MA e } \\
\text { Bevilacqua MC }^{5}\end{array}$ & $\begin{array}{l}\text { Uso Alternativo do Sistema } \\
\text { de Freqüência Modulada (FM): Crianças } \\
\text { com Dificuldades de Aprendizagem } \\
\text { e Déficit de Atenção }\end{array}$ & 2002 & estudo de Casos & 5 \\
\hline Weihing $\mathrm{J}^{22}$ & FM systems as a treatment for CAPD & 2005 & opinião de especialista & 6 \\
\hline Whitelaw $\mathrm{GM}^{25}$ & FM candidacy issues and the "alphabet soup" & 2004 & opinião de especialista & 6 \\
\hline Wertz D et al ${ }^{21}$ & $\begin{array}{c}\text { Auditory Processing Disorders: Management Approaches Past to } \\
\text { Present }\end{array}$ & 2002 & opinião de especialista & 6 \\
\hline Young et all ${ }^{2}$ & $\begin{array}{l}\text { Preferred FM system listening levels of children with central } \\
\text { auditory processing disorders. }\end{array}$ & 1997 & $\begin{array}{l}\text { estudos descritivos } \\
\text { (estudo coorte) }\end{array}$ & 4 \\
\hline
\end{tabular}


Os estudos clínicos randomizados controlados são descritos como o "padrão-ouro" na avaliação de questões terapêuticas em saúde já que por meio deste tipo de estudo reduz-se a probabilidade de obter dados tendenciosos (viés) na pesquisa, privilegiando assim a qualidade da informação.

As informações dos autores, título, ano, nível de evidência para os 20 trabalhos recuperados encontram-se na Tabela 4. Tendo como base as recomendações Consort nenhum dos trabalhos foi classificado como sendo estudo clínico randomizado controlado.

Pode-se verificar que catorze trabalhos (70\%) foram classificados como "opinião de especialistas", três (15\%) como "estudo de casos" e três "estudos descritivos" (15\%). Tais desenhos de estudo apresentam evidência científica baixa.

\section{Conclusão}

Um dos objetivos principais de uma revisão sistemática é agregar fortes evidências de pesquisa para guiar a prática clínica. A inexistência de estudos clínicos randomizados controlados que avaliem a efetividade do sistema FM pessoal na intervenção de indivíduos com transtorno de processamento auditivo central limita as considerações do presente trabalho.

A execução dos estudos clínicos randomizados controlados é tida como complexa e de custo elevado, fazendo com que outros tipos de estudos experimentais sejam utilizados para fundamentar práticas em saúde. Embora no presente trabalho não tenha havido um esforço exaustivo para recuperar todas as publicações não indexadas (por exemplo, anais de congressos) chama a atenção que nem mesmo estes outros tipos de estudos foram encontrados em abundância e a indicação do sistema FM no transtorno de processamento auditivo central parece derivar quase que totalmente da opinião de especialistas (Tabela 4).

Embora os conhecimentos básicos aprendidos em livros e com especialistas mais experientes sejam essenciais para a prática clínica, estes não podem ser a única base para tomada de decisões clínicas ${ }^{8-}$ ${ }^{9}$, sobretudo na época atual, onde se preconiza a oferta de serviços de qualidade associada ao uso racional de recursos, sejam estes públicos ou privados.

Para crianças com TPA vem sendo recomendado o uso de uma tecnologia que proporcione audibilidade e clareza do sinal acústico, como, por exemplo, o sistema $\mathrm{FM}^{12-21}$ sendo este considerado parte do processo de reabilitação e não a única estratégia para o mesmo ${ }^{22}$. Porém poucos dados científicos que demonstrem a eficácia do sistema FM como uma estratégia de reabilitação de pessoas com TPA foram publicados ${ }^{23-29}$.

Embora seja possível que o sistema FM seja um recurso efetivo no tratamento dos TPA, à luz das publicações analisadas a presente revisão não pode confirmar ou excluir tal possibilidade. Uma vez que este equipamento é freqüentemente indicado na prática clínica justifica-se a necessidade de realização de estudos clínicos randomizados controlados comparando o uso do sistema FM a outras formas de tratamento, ou até mesmo, à ausência de algum tipo de tratamento - sempre e quando o design do estudo não implique em infringir princípios éticos. Caso seja encontrada alguma evidência de efetividade do sistema FM sobre outras formas de intervenção, convém comparar os tipos de FM disponíveis (pessoal versus campo livre). Finalmente é de importância também considerar a perspectiva do usuário ou de sua família/cuidadores.

Agradecimentos: à bibliotecária Cybelle de Assumpção Fontes, Diretora Técnica do Serviço de Biblioteca e Documentação Professor Doutor Antônio Gabriel Atta da Faculdade de Odontologia de Bauru - USP, pela orientação e auxílio nos procedimentos de busca e recuperação das referências. Os autores agradecem à Beatriz Tangerino Junqueira pela tradução do artigo para o Inglês. 


\section{Referências Bibliográficas}

1. Momensohn-Santos TM, Branco-Barreiro FCA. Avaliação e intervenção fonoaudiológica no transtorno de processamento auditivo. In: Ferreira LP, Befi-Lopes DM, Limongi SCO. Tratado de Fonoaudiologia. São Paulo: Roca; 2004. p. 553-68.

2. ASHA: American Speech-Language-Hearing Association. (Central) auditory processing disorders - the role of the audiologist [Position statement], 2005a. [Acesso em 16 nov 2005]. Disponível em URL: www.asha.org/members/ deskref-journals/deskref/default.

3. Dreossi RCF, Momensohn-Santos TM. Investigação sobre o efeito do ruído na inteligibilidade de fala de crianças da quarta série do ensino fundamental. Rev Soc Bras Fonoaudiol. 2004;9(3):136-43.

4. Blasca WQ, Ferrari DV, Jacob RTS. Dispositivos Eletrônicos Aplicados à surdez: conceitos básicos. In: Genaro KF, Lamonica DC, Bevilacqua MC (org). O processo de comunicação: contribuição para a formação de professores na inclusão de indivíduos com necessidade educacionais especiais. São José dos Campos: Pulso; 2006, cap 14. p. 197-213.

5. Souza-Jacob RT, Almeida MA e Bevilacqua MC. Uso Alternativo do Sistema de Freqüência Modulada (FM): Crianças com Dificuldades de Aprendizagem e Déficit de Atenção. J Bras Fonoaudiol. 2002;3(10):54-9.

6. Pfeffer EB. Alternate uses for FM systems. In: Ross, M. FM auditory systems- characteristics, selection and use. Timonium: York Press; 1992, cap 10. p. 211-23.

7. Chermak GD, Musiek F. Central auditory processing disorders - new perspectives. San Diego: Singular Publishing Group; 1997.

8. Dollaghan CA. Evidence-based practice in communication disorders: what do we know, and when do we know it? J Communic Disord. 2004;37:391-400.

9. Cox RM. Evidence-based practice in provision of amplification. J Am Acad Audiol. 2005;16(7):419-38.

10. El Dib RP, Atallah AN. Evidence-based speech, language and hearing therapy and the Cochrane Library's systematic reviews. Sao Paulo Med J. 2006;124(2):51-4.

11. Moher D, Schultz KF, Altman DG. The CONSORT statement: revised recommendations for improving the quality of reports of parallel-group randomized trials. Annals Intern Medic. 2001;134(8):657-62.

12. Young D, Bradle P, Hickson L, Lawson M. Preferred FM system listening levels of children with central auditory processing disorders. J Acad Rehab Audiol. 1997;30:53-61.

13. Bellis TJ. Developing deficit-specific intervention plans for individuals with auditory processing disorders. Semin Hear. 2002;23(4):287-95.

14. Friel-Patti S. Clinical Decision-Making in the Assessment and Intervention of Central Auditory Processing Disorders. Lang Speech Hearing Services Schools. 1999;30:345-52.
15. Launer S. Wireless solutions: the state of the art and future of technology for the hearing impaired consumer. In: Achieving Clear Communication Employing Sound Solutions 2003: Proceedings of the First International Conference. Chicago: Phonak AG; 2004. p. 31-8. [Acesso em 16 nov 2005]. Disponível em URL: http:// Www.phonak.dk/com_2003proceedings_chapter1.pdf.

16. Baran JA. Managing auditory processing disorders in adolescents and adults. Semin Hear. 2002;23:327-36.

17. Ferre JM. Managing children's central auditory processing deficits in the real world: what teachers and parents want to know. Semin Hear. 2002;23:319-26.

18. Beck DL, Bellis TJ. (Central) auditory processing disorders: overview and amplification issues. Hear J. 2007;60(5):44-7.

19. Flexer C. The impact of classroom acoustics: listening, learning, and literacy. Semin Hear. 2004;25(2):131-40.

20Rosenberg GG. Classroom acoustics and personal FM technology in managemet of auditory processing disorder. Semin Hear. 2002;23(4):309-17.

21. Wertz D , Hall JW, Davis W II. Auditory processing disorders: management approaches past to present. Semin Hear. 2002;23:277-86.

22. Weihing J. FM systems as a treatment for CAPD. Hear J. 2005;58(10):74.

23. Keith RW. Clinical issues in central auditory processing disorders. Lang Speech Hearing Services Schools. 1999;30:339-44 [Acesso em: 19 nov 2005]. Disponível em URL: http://www.asha.org/NR/rdonlyres/3D9D9107A934-4077-9FE7-5732C0120C50/0/11069_1.pdf

24. ASHA: American Speech-Language-Hearing Association. (Central) auditory processing disorders. 2005b. [Acesso em: 16 nov 2005]. Disponível em URL: www.asha.org/members/deskref-journals/deskref/default.

25. Whitelaw GM, FM Candidacy issues and the "alphabet soup". In: Achieving Clear Communication Employing Sound Solutions 2003: Proceedings of the First International Conference. Chicago: Phonak AG; 2004. p.101-106. [Acesso em: 16 nov 2005]. Disponível em URL: http:// www.phonak.dk/com_2003proceedings_chapter8.pdf.

26. Kreisman BM, Crandell CC. Frequency Modulation (FM) Systems for children with normal hearing. [Acessso em 02 de março de 2208]. Disponível em URL: http:// w w w. A u d i o log y o n li n e.com/a r t i c le s/ pf_article_detail.asp?article_id=358.

27. Phonak. Ear-level FM receiver stimulates auditory neural plasticity in children with APD. Field Study News; march 2006. 44 [Acesso em: 19 nov 2005]. Disponível em URL: http://www.speechpathology.com/channels/ phonak_field_06march.pdf.

28. Sharma M, Purdy SC. A case study of an 11-year-old with auditory processing disorder. Aust \& NZ J Audiol. 2007;29(1):40-52.

29. Bellis TJ, Ferre MF. Multidimensional approach to the differential diagnosis of central auditory processing disorders in children. J Am Acad Audiol. 1999;10:319-28.

30. Cameron S, Dillon H. The listening in spatialized noise-sentences test (LISN-S): test-retest reliability study. Int J Audiol. 2007;46(3):145-53. 